\title{
Assessment of bronchial and pulmonary blood supply in non-small cell lung cancer subtypes using computed tomography perfusion
}

\author{
Nguyen-Kim, Thi Dan Linh ; Frauenfelder, Thomas ; Strobel, Klaus ; Veit-Haibach, Patrick ; Huellner,
} Martin W

\begin{abstract}
OBJECTIVES: The aim of this study was to investigate the dual blood supply of non-small cell lung cancer (NSCLC) and its association with tumor subtype, size, and stage, using computed tomography perfusion (CTP). MATERIALS AND METHODS: A total of 54 patients (median age, 65 years; range, 42-79 years; 15 women, 39 men) with suspected lung cancer underwent a CTP scan of the lung tumor. Pulmonary and bronchial vasculature regions of interest were used to calculate independently CTP parameters (blood flow $[\mathrm{BF}]$, blood volume $[\mathrm{BV}]$, and mean transit time $[\mathrm{MTT}]$ ) of the tumor tissue. The mean and maximum pulmonary and bronchial perfusion indexes (PImean and PImax) were calculated. The tumoral volume and the largest tumoral diameter were assessed. Differences in CTP parameters and indexes among NSCLC subtypes, tumor stages and tumor dimensions were analyzed using non-parametric tests. RESULTS: According to biopsy, 37 patients had NSCLC (22 adenocarcinomas [ACs], 8 squamous cell carcinomas [SCCs], 7 large-cell carcinomas [LCC]). The mean bronchial BF/pulmonary BF, bronchial BV/pulmonary BV, and bronchial MTT/pulmonary MTT was 41.2 \pm 30.0/36.9 $\pm 24.2 \mathrm{~mL} / 100 \mathrm{~mL} / \mathrm{min}, 11.4 \pm 9.7 / 10.4 \pm 9.4 \mathrm{~mL} / 100 \mathrm{~mL}$, and $11.4 \pm 4.3 / 14.9 \pm 4.4$ seconds, respectively. In general, higher bronchial BF than pulmonary BF was observed in NSCLC $(\mathrm{P}=0.014)$. Using a tumoral volume cutoff of $3.5 \mathrm{~cm}$, a significant difference in pulmonary PImax was found $(\mathrm{P}=$ 0.028). There was a significantly higher mean pulmonary BF in LCCs and SCCs compared with ACs ( $P$ $=0.018$ and $\mathrm{P}=0.044$, respectively), whereas the mean bronchial $\mathrm{BF}$ was only significantly higher in LCCs compared with ACs $(\mathrm{P}=0.024)$. Correspondingly, the PImax was significantly higher in LCCs and SCCs than in ACs $(\mathrm{P}=0.001$ for both). Differences between bronchial and pulmonary PImean and PImax among $\mathrm{T}$ stages and Union Internationale Contre le Cancer stages were not statistically significant ( $\mathrm{P}$ values ranging from 0.691 to 0.753 ). CONCLUSIONS: The known dual blood supply of NSCLC, which depends on tumor size and histological subtype, is reflected in CTP parameters, with parameters depending both on tumor size and histological subtype. This has to be accounted for when analyzing NSCLC with CTP.This is an open-access article distributed under the terms of the Creative Commons Attribution-NonCommercial-NoDerivatives 3.0 License, where it is permissible to download and share the work provided it is properly cited. The work cannot be changed in any way or used commercially. http://creativecommons.org/licenses/by-nc-nd/3.0.
\end{abstract}

DOI: https://doi.org/10.1097/RLI.0000000000000124

Posted at the Zurich Open Repository and Archive, University of Zurich

ZORA URL: https://doi.org/10.5167/uzh-103173

Journal Article

Published Version 
Originally published at:

Nguyen-Kim, Thi Dan Linh; Frauenfelder, Thomas; Strobel, Klaus; Veit-Haibach, Patrick; Huellner, Martin W (2015). Assessment of bronchial and pulmonary blood supply in non-small cell lung cancer subtypes using computed tomography perfusion. Investigative Radiology, 50(3):179-186.

DOI: https://doi.org/10.1097/RLI.0000000000000124 


\title{
Assessment of Bronchial and Pulmonary Blood Supply in Non-Small Cell Lung Cancer Subtypes Using Computed Tomography Perfusion
}

\author{
Thi Dan Linh Nguyen-Kim, MD, * Thomas Frauenfelder, MD, * Klaus Strobel, MD, $\uparrow$ \\ Patrick Veit-Haibach, MD, ${ }^{*} \neq$ and Martin W. Huellner, MD $\$$
}

\begin{abstract}
Objectives: The aim of this study was to investigate the dual blood supply of non-small cell lung cancer (NSCLC) and its association with tumor subtype, size, and stage, using computed tomography perfusion (CTP).

Materials and Methods: A total of 54 patients (median age, 65 years; range, 42-79 years; 15 women, 39 men) with suspected lung cancer underwent a CTP scan of the lung tumor. Pulmonary and bronchial vasculature regions of interest were used to calculate independently CTP parameters (blood flow [BF], blood volume [BV], and mean transit time [MTT]) of the tumor tissue. The mean and maximum pulmonary and bronchial perfusion indexes $\left(\mathrm{PI}_{\text {mean }}\right.$ and $\left.\mathrm{PI}_{\max }\right)$ were calculated. The tumoral volume and the largest tumoral diameter were assessed. Differences in CTP parameters and indexes among NSCLC subtypes, tumor stages and tumor dimensions were analyzed using non-parametric tests.

Results: According to biopsy, 37 patients had NSCLC (22 adenocarcinomas [ACs], 8 squamous cell carcinomas [SCCs], 7 large-cell carcinomas [LCC]). The mean bronchial BF/pulmonary BF, bronchial BV/pulmonary BV, and bronchial MTT/pulmonary MTT was $41.2 \pm 30.0 / 36.9 \pm 24.2 \mathrm{~mL} / 100 \mathrm{~mL} / \mathrm{min}$, $11.4 \pm 9.7 / 10.4 \pm 9.4 \mathrm{~mL} / 100 \mathrm{~mL}$, and $11.4 \pm 4.3 / 14.9 \pm 4.4$ seconds, respectively. In general, higher bronchial BF than pulmonary BF was observed in NSCLC $(P=0.014)$. Using a tumoral volume cutoff of $3.5 \mathrm{~cm}^{3}$, a significant difference in pulmonary $\mathrm{PI}_{\max }$ was found $(P=0.028)$. There was a significantly higher mean pulmonary BF in LCCs and SCCs compared with ACs $(P=$ 0.018 and $P=0.044$, respectively), whereas the mean bronchial BF was only significantly higher in LCCs compared with ACs $(P=0.024)$. Correspondingly, the $\mathrm{PI}_{\max }$ was significantly higher in LCCs and SCCs than in ACs $(P=0.001$ for both). Differences between bronchial and pulmonary $\mathrm{PI}_{\text {mean }}$ and $\mathrm{PI}_{\max }$ among $\mathrm{T}$ stages and Union Internationale Contre le Cancer stages were not statistically significant ( $P$ values ranging from 0.691 to 0.753 ).

Conclusions: The known dual blood supply of NSCLC, which depends on tumor size and histological subtype, is reflected in CTP parameters, with parameters depending both on tumor size and histological subtype. This has to be accounted for when analyzing NSCLC with CTP.
\end{abstract}

Key Words: computed tomography, perfusion, lung neoplasms, multimodal imaging, medical oncology

(Invest Radiol 2015;00: 00-00)

hroughout the world, lung cancer still accounts for more deaths than any other cancer does. More than 200,000 new cases and

Received for publication August 18, 2014; and accepted for publication, after revision, October 22, 2014

From the *Institute of Diagnostic and Interventional Radiology, University Hospital Zurich, Zurich; †Institute of Radiology and Nuclear Medicine, Lucerne Cantonal Hospital, Lucerne; and †Division of Nuclear Medicine and §Clinic of Neuroradiology, University Hospital Zurich, Zurich, Switzerland.

Conflicts of interest and sources of funding: none declared.

Reprints: Thi Dan Linh Nguyen-Kim, MD, Institute of Diagnostic and Interventional Radiology, University Hospital Zurich, Switzerland, Rämistrasse 100, CH-8091 Zurich, Switzerland. E-mail: thidalinh.nguyen@usz.ch.

Copyright (C) 2014 by Lippincott Williams \& Wilkins

ISSN: 0020-9996/15/0000-0000 approximately 160,000 deaths, accounting for more than one quarter of all cancer-related deaths, are expected in the United States for 2014. ${ }^{1}$ Non-small cell lung cancer (NSCLC) constitutes most of lung cancer. In most patients, the disease is already advanced at the time of the initial diagnosis, and hence, palliative radiation therapy and chemotherapy are initiated as first-line regimens. ${ }^{2,3}$ The proliferation of new blood vessels, originating from the existing vascular system, is needed both for tumor growth and metastatic dissemination. ${ }^{4-6}$ Monoclonal antibodies, such as bevacizumab, targeting vascular endothelial growth factor to inhibit tumoral angiogenesis, suppress tumor growth. Such targeted drugs are increasingly used in combination with standardized platinum-based chemotherapy. ${ }^{2,7}$ Patient treatment hence becomes more individualized to tumor biology, taking into account angiogenic status, which still needs to be learned and understood. ${ }^{7,8}$ The traditional monitoring of therapeutic effects, being based on morphological criteria on computed tomography (CT), may lead to a misinterpretation of antiangiogenic effects. ${ }^{9,10}$ Hence, the evolving therapeutic field of vascular-modulating drugs requires new noninvasive methods for assessing tumor vascularity in vivo. ${ }^{11-15}$

In 1967, Milne ${ }^{16}$ showed that lung tumors may have a dual blood supply by the pulmonary and the aortic system, with a trend toward histological type-specific circulatory pattern. The advent of CT perfusion (CTP) within the last decade allowed for a direct, noninvasive quantification of the vascular function. ${ }^{17,18}$ Functional parameters, derived from the assessment of peak tumor enhancement over time in the tissue during the CTP scan, give information about blood volume (BV), blood flow (BF), and mean transit time (MTT) within the network of tumoral neovascularity, as well as the rate of abnormal leakage into the extracellular space, due to the vascular hyperpermeability in the abnormal neovessel wall. ${ }^{5,19}$ One of the first applications for the assessment of lung lesions with CTP was pulmonary nodules, particularly in comparison with ${ }^{18} \mathrm{~F}$-fluoro-2-deoxy-D-glucose positron emission tomography/ $\mathrm{CT}$ (PET/CT) ${ }^{20}$ Cutoff values have been defined for several CTP parameters to differentiate benign from malignant pulmonary nodules, with lower perfusion index (PI) and higher BF and BV obtained in malignancy. ${ }^{21,22}$ Similar accuracy of CTP and fluoro-2-deoxy-D-glucose$\mathrm{PET} / \mathrm{CT}$ was confirmed in the meta-analysis by Cronin et al. ${ }^{23}$ Several studies have addressed differences of CTP parameters among histological lung cancer subtypes, assessing them with a single-input CTP analysis of either the pulmonary or the bronchial arterial system. ${ }^{24-27}$ Computed tomography perfusion in lung cancer remains a technical challenge, particularly due to breathing motion over time. ${ }^{28}$ Furthermore, a true separation of pulmonary and bronchial BF using a CTP analysis is technically not possible at this time because blood from both vascular inputs is mixed within lesions at a microscopic level, precluding both spatial and temporal separation. Despite these difficulties, Yuan et $\mathrm{al}^{22}$ recently demonstrated that a dual-input maximum slope CTP analysis is valuable in NSCLC. However, many centers do not fulfill the hardware and software requirements for such a dual-input analysis and rely on either single-input pulmonary or bronchial CTP assessment. Currently, there is no universally accepted consensus 
regarding into which vascular system the arterial input region of interest (ROI) should be placed, ${ }^{27,29,30}$ and either method's results are used interchangeably. The differences in pulmonary circulation-derived and bronchial circulation-derived analyses are still unclear, and a direct comparison of CTP parameters derived with these two different methods is problematic. Besides, information about the association of pulmonary-derived and bronchial-derived CTP parameters with tumor size, volume, and stage in different NSCLC subtypes is still limited.

We hypothesized that values of CTP parameters obtained by pulmonary or bronchial analysis might be different depending on tumor type, stage, and size. Such differences would have to be accounted for analyzing NSCLC using CTP with either vasculature approach in a clinical setup. Thus, the purpose of this study was to investigate the relevance of pulmonary and bronchial ROI placement in the CTP assessment of dually blood-supplied NSCLC and its association with histological subtypes, tumor size, and tumor stage.

\section{MATERIALS AND METHODS}

\section{Study Population}

A total of 54 patients (median age, 61 years; range, 42-79 years; 15 women, 39 men) with suspected lung cancer being referred to our $\mathrm{PET} / \mathrm{CT}$ center for baseline staging were prospectively enrolled in this ethical committee-approved study between January 2012 and December 2012. All patients provided signed informed consent before the examinations. All subjects underwent a CTP scan of the suspected lung tumor. Exclusion criteria were unwillingness to undergo the CTP scan, renal insufficiency in nondialyzed patients, pregnancy, untreated hyperthyroidism, severe respiratory dysfunction, and known previous reaction after administration of iodinated contrast medium. A definitive histopathological diagnosis was obtained in all subjects by CT-guided biopsy, bronchofibroscopic biopsy, or tumor resection (median time interval, 6 days after CTP scan; range, $0-18$ days). Thirteen patients were excluded after the scan because histology results revealed lesions other than NSCLC (small cell lung cancer, 5 patients; inflammatory myofibroblastic pseudotumor, 1 patient; cavitating staphylococcal pneumonia, 1 patient; streptococcal pneumonia, 1 patient; pulmonary sequester, 1 patient; lymphoma, 1 patient; sarcoma, 1 patient; pulmonary hamartoma, 1 patient; and tularemia, 1 patient). In 4 patients, the subsequent combined pulmonary and bronchial CTP analysis was not possible because of technical problems. Finally, 37 patients were eligible for analysis. The NSCLC subtype, tumor size, volume, and stage were recorded in all patients. The standard of reference consisted of postsurgical histopathology results in 13 subjects, providing of pathological tumor stage. In the remaining 24 patients, biopsy-derived histopathology and clinical tumor stage were used.

\section{CT Perfusion Protocol}

Patients were advised to resume shallow breathing for the entire duration of the scan. All scans were performed on a 256-slice CT scanner (Somatom Definition Flash, Siemens Healthcare, Erlangen, Germany). The $Z$-axis scanning length was $7 \mathrm{~cm}$. This relatively large scan length was chosen to cover the whole tumor as well as major pulmonary and bronchial vessels in every patient. The tube current was fixed to 100 $\mathrm{mAs}$, and the tube voltage set to $100 \mathrm{kV}$. An automatic exposure control was not used. The duration of the whole CTP scan was 60 seconds, with a rotation time of 1 second, and 1 scan per second. The volume CT dose index and dose-length product were $193.1 \mathrm{mGy}$ and $1707 \mathrm{mGy} \mathrm{cm}$, respectively, corresponding to a calculated effective dose of $23.9 \mathrm{mSv}$. The CTP scanning was started with a delay of 3 seconds after the initiation of the injection of $40 \mathrm{~mL}$ of iodinated contrast medium (Ultravist 370 , Bayer Healthcare, Leverkusen, Germany) at a flow rate of $4.5 \mathrm{~mL} / \mathrm{s}$. The contrast medium was injected into an antecubital vein by a dualhead pump injection device (Stellant D, Medrad, Warrendale, PA), followed by a flush of $50 \mathrm{~mL}$ of saline at $4.5 \mathrm{~mL} / \mathrm{s}$. This rather lengthy protocol was chosen to ensure a high reproducibility of perfusion parameters. ${ }^{31}$ The slice-collimation was set to $64 \times 0.6 \mathrm{~mm}$. The reconstruction increment was $3 \mathrm{~mm}$ with a $5-\mathrm{mm}$ slice width. Image reconstruction was performed with a $512 \times 512$ pixel matrix and medium smooth B30f kernel.

\section{Data Postprocessing and Image Analysis}

For postprocessing and analysis, the reconstructed images were transferred to a commercially available computer workstation with dedicated perfusion software (Leonardo; Syngo Volume Perfusion CT Body, Siemens Healthcare). All procedures were carried out by 1 boardcertified radiologist (D.N.) with 6 years of experience in chest imaging. After computerized motion correction, a semiautomatic freehand segmentation of the tumor tissue volume over time was performed. To investigate the pulmonary and bronchial CTP parameters independently, an elliptical ROI was placed in the pulmonary trunk and in the descending aorta at the level of the bronchial arteries, respectively. If the pulmonary trunk was not covered by the CTP volume, the left or right pulmonary artery was chosen. Time density curves of tissue with different peak times were calculated, depending on whether a pulmonary-based or bronchialbased examination was performed.

Pulmonary and bronchial CTP parameters, including specifically the mean BF, BV, and MTT of the tumor tissue, as well as the maximum $\mathrm{BF}\left(\mathrm{BF}_{\max }\right)$, were calculated automatically by the software (Figs. 1-3). The mean PI ( $\left.\mathrm{PI}_{\text {mean }}\right)$ and the maximum PI $\left(\mathrm{PI}_{\max }\right)$ for both the pulmonary and bronchial blood circulation were calculated according to the following formulas:

pulmonaryP $\mathrm{I}_{\text {mean }}=(($ pulmonary $B F) /($ pulmonary $B F+$ bronchial $B F))$

pulmonaryP $_{\max }=(($ pulmonary BF $\max ) /($ pulmonary $B F \max +$ bronchial $B F \max ))$,

bronchial $\mathrm{PI}_{\text {mean }}=\left(\frac{\text { bronchial } B F}{\text { pulmonary } B F+\text { bronchial BF }}\right)$, and

bronchialPI $_{\max }=\left(\frac{\text { bronchial BFmax }}{\text { pulmonary BF } \max +\text { bronchial BF } \max }\right)$

The longest diameter of the tumor was determined based on the morphological CT data set. The tumor volume was segmented semiautomatically using a dedicated volumetry software (Myrian, Intrasense, Paris, France).

\section{Statistical Analysis}

All statistical analyses were performed using IBM SPSS Statistics 19.0.1 (SPSS Inc, Chicago, IL). Differences in pulmonary circulationderived CTP parameters and bronchial circulation-derived CTP parameters and indexes were analyzed using the Wilcoxon signed-ranks test. Differences in CTP parameters and indexes among NSCLC subtypes, tumor stages, and among small and large tumors were analyzed using the Wilcoxon signed-ranks test or the Kruskal-Wallis test. Correlation analyses were performed using Spearman correlation coefficient to analyze the relationship of CTP parameters and indexes with the tumor diameter, tumor volume, T stage, and Union Internationale Contre le Cancer stage.

\section{RESULTS}

Histology results revealed that our study cohort consisted of 22 adenocarcinomas (ACs), 8 squamous cell carcinomas (SCCs), and 7 large-cell carcinomas (LCCs). During therapy, 13 patients (8 with AC, 4 with SCC, 1 with LCC) underwent curative surgery and adjuvant radiochemotherapy, providing pathological TNM status analysis. Thirteen patients underwent primary curative radiochemotherapy; 10, palliative 


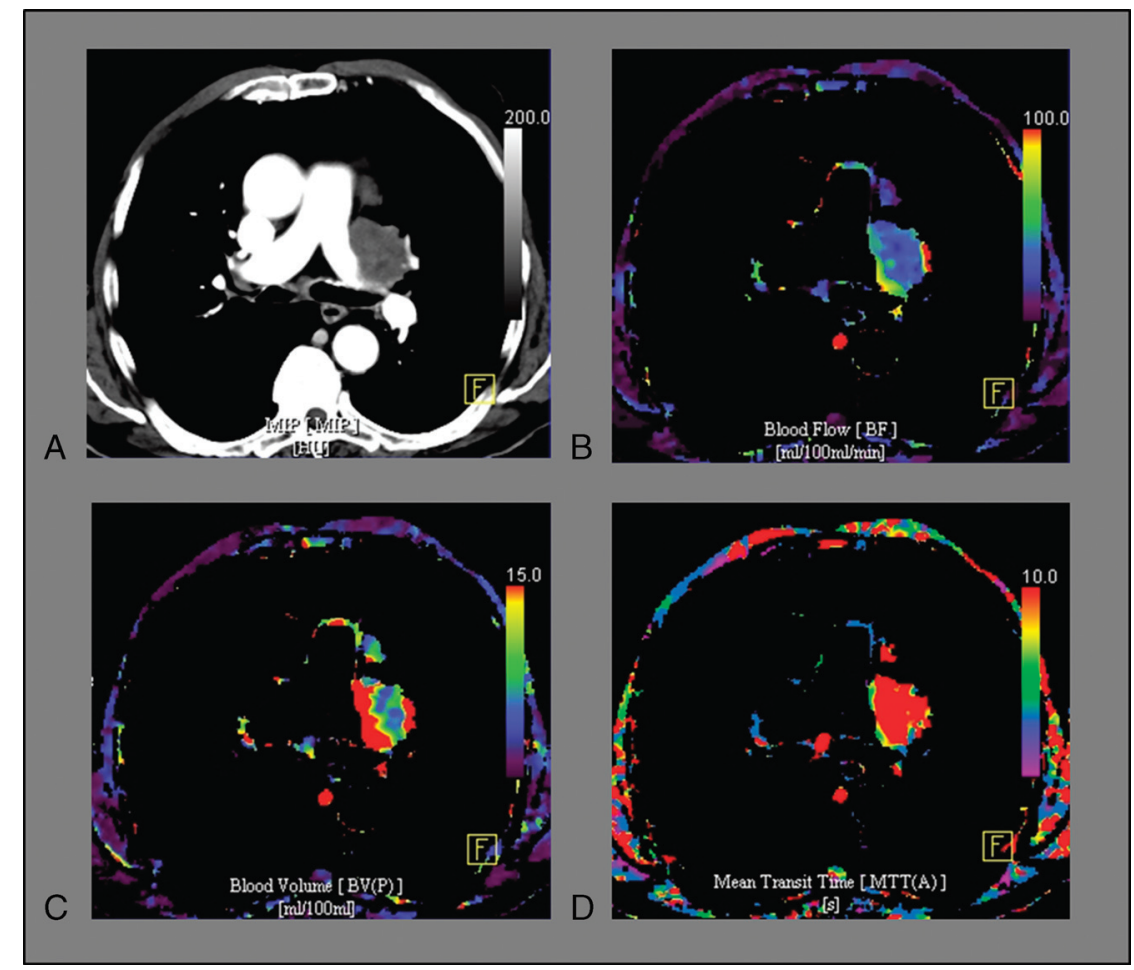

FIGURE 1. Squamous cell carcinoma of the left upper lobe assessed using a bronchial vasculature approach. The MIP of computed tomography perfusion over time (A) and color-coded parametric maps for BF (B), BV (C), and MTT (D) are shown. BF indicates blood flow; BV, blood volume; MIP, maximum intension projection; MTT, mean transit time.

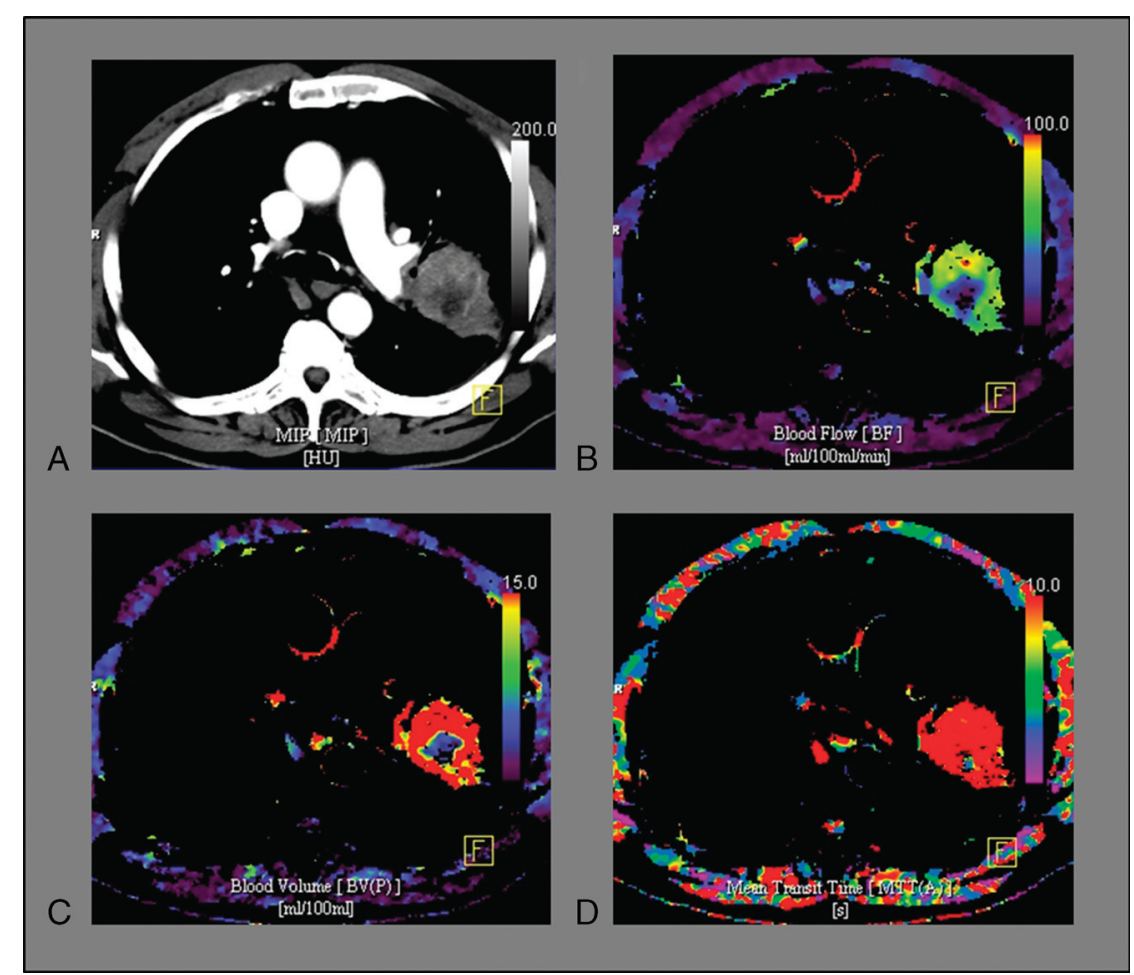

FIGURE 2. Adenocarcinoma of the left upper lobe assessed using a bronchial vasculature approach. The MIP of computed tomography perfusion over time (A) and color-coded parametric maps for BF (B), BV (C), and MTT (D) are shown. BF indicates blood flow; BV, blood volume; MIP, maximum intension projection; MTT, mean transit time. 


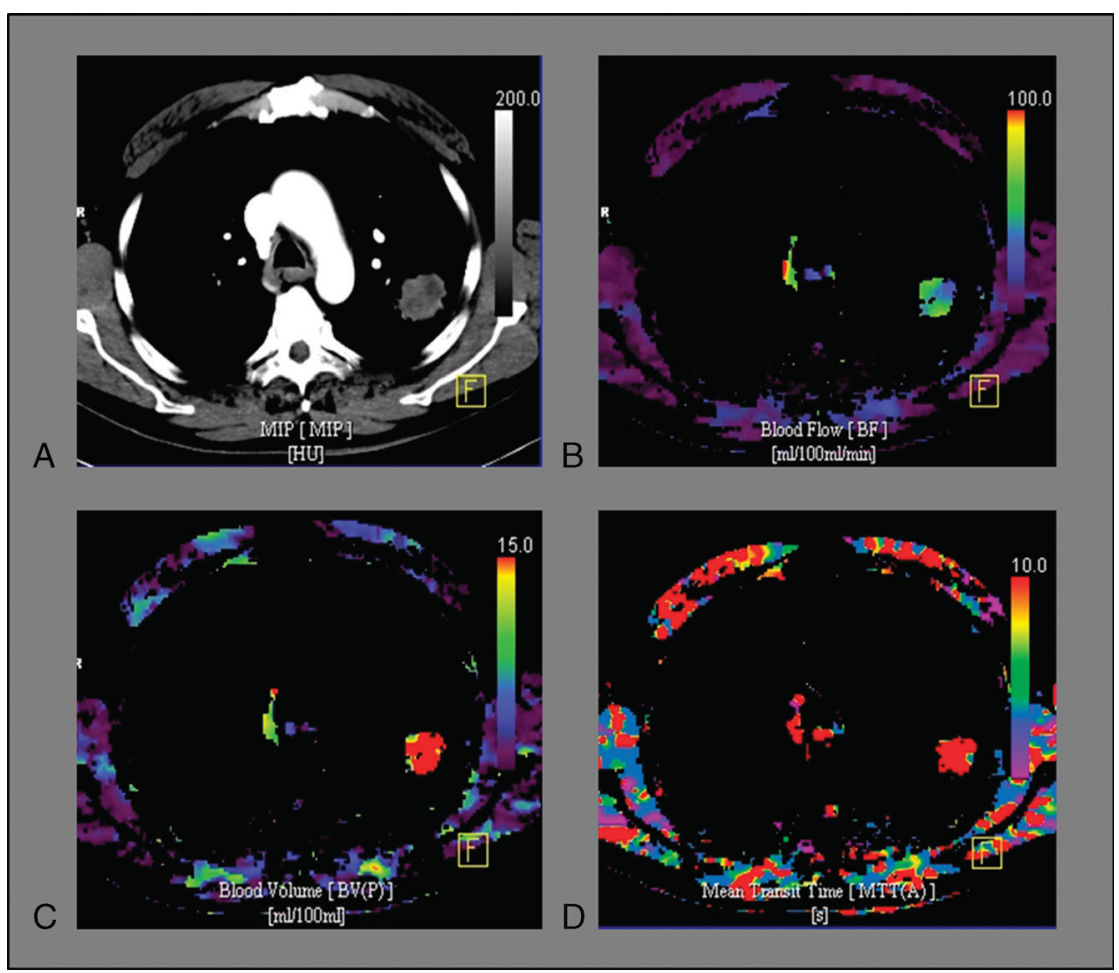

FIGURE 3. Squamous cell carcinoma of the left upper lobe assessed using a bronchial vasculature approach. The MIP of computed tomography perfusion over time (A) and color-coded parametric maps for BF (B), BV (C), and MTT (D) are shown. BF, blood flow; BV, blood volume; MIP, maximum intension projection; MTT, mean transit time.

chemotherapy; and 1, neoadjuvant therapy and surgery. In these 24 patients, only clinical TNM was available. The tumor stage was T1 in 4 patients, T2 in 25 patients, T3 in 4 patients, and T4 in 4 patients. More detailed characteristics of the study population are given in Table 1 .

For all tumors, the mean pulmonary/bronchial BF was 36.9/41.2 $\mathrm{mL} / 100 \mathrm{~mL} / \mathrm{min}$, the maximum pulmonary/bronchial BF was $56.4 / 64.6$ $\mathrm{mL} / 100 \mathrm{~mL} / \mathrm{min}$, the mean pulmonary/bronchial BV was $10.4 / 11.4$ $\mathrm{mL} / 100 \mathrm{~mL}$, and the mean pulmonary/bronchial MTT was 14.9/ 11.4 seconds.

Overall, the bronchial circulation-derived $\mathrm{BF}$ was significantly higher than the pulmonary circulation-derived BF $(P=0.014)$ (Table 2). Within the subanalysis of histological subtypes of NSCLC, only the $\mathrm{BF}_{\max }$ of $\mathrm{AC}$ was different depending on the type of assessment, albeit with borderline significance $(P=0.049)$ (Table 2). Such a trend toward higher bronchial than pulmonary BF could also be seen for the remaining subgroups SCC and LCC, however, without reaching statistical significance ( $P=0.401$ and $P=0.237$, respectively). The significant differences in MTT measured with both methods $(P$ values ranging from $<0.001$ to 0.018 ) are a direct consequence of our approach and are listed in Table 2 only for the sake of completeness. A detailed overview of CTP parameters of NSCLC subtypes is given in Table 3. Pulmonary- and bronchial-derived BFs were both significantly higher in LCC than in AC ( $P=0.018, P=0.024$, respectively). Pulmonary $\mathrm{PI}_{\max }$ was significantly higher in LCC and SCC than in AC $(P=0.001$, each) (Fig. 4). Differences in $\mathrm{PI}_{\text {mean }}$ and $\mathrm{PI}_{\max }$ among $\mathrm{T}$ stages and Union Internationale Contre le Cancer stages were statistically not significant ( $P$ values ranging from 0.691 to 0.753 ), as well as differences between other CTP parameters. Using a volume threshold of $3.5 \mathrm{~cm}^{3}$ (tumors $<3.5 \mathrm{~cm}^{3}, \mathrm{n}=15$; tumors $>3.5 \mathrm{~cm}^{3}, \mathrm{n}=22$ ), the pulmonary $\mathrm{PI}_{\max }$ was found to be significantly different $(P=0.028)$ (Fig. 5), whereas no differences were found for the bronchial $\mathrm{BF}_{\max }$ and pulmonary $\mathrm{BF}_{\max }(P=0.646$, $P=0.805$, respectively). Furthermore, no discriminative threshold could be found for the tumor diameter.

\section{DISCUSSION}

It is known that NSCLCs have a dual blood supply, with contributions both from the pulmonary and the bronchial vasculature. ${ }^{16}$ Consistent with the results of the recent study by Yuan et al, ${ }^{22}$ our findings show that, in NSCLC, the bronchial contribution is higher than the pulmonary contribution. In addition, we have now demonstrated that there are also differences depending on the NSCLC subtype: LCC shows higher bronchial and pulmonary $\mathrm{BV}$ and $\mathrm{BF}$ compared with $\mathrm{AC}$ and SCC. And the pulmonary $\mathrm{PI}_{\max }$ was found to be significantly higher in LCC and SCC compared with AC.

In general, higher perfusion is believed to be related to more aggressive tumor features, such as local invasiveness and metastatic capability. Our results likely reflect the biological nature of LCC, which is known to be a poorly differentiated tumor and clinically often shows a similar behavior as small cell lung cancer, concerning its aggressiveness and poor long-term survival. ${ }^{2-35}$ Correspondingly, Fraioli and

TABLE 1. Characteristics of the Study Cohort $(n=37)$

\section{Parameter}

Age, median (range), y

Sex ratio (female/male)

68 (51-79)

BMI, median (range), $\mathrm{kg} / \mathrm{m}^{2}$

$8 / 29$

Tumor volume, median (range), $\mathrm{cm}^{3}$

$26.1(19.5-29.4)$

Tumor diameter, median (range), $\mathrm{cm}$

T stage, median (range)

$13.2(0.8-156)$

$2.78(1-50.9)$

$2(1-4)$

III (I-IV)

BMI indicates body mass index; UICC, Union Internationale Contre le Cancer. 
TABLE 2. CT Perfusion Parameters in All Tumors, Adenocarcinomas, Squamous Cell Carcinomas, and Large-Cell Carcinomas, Stratified by Pulmonary and Bronchial Vasculature Assessment

\begin{tabular}{|c|c|c|c|}
\hline Parameter & Pulmonary & Bronchial & $P$ \\
\hline \multicolumn{4}{|l|}{ All tumors $(\mathrm{n}=37)$} \\
\hline $\mathrm{BF}$, mean $\pm \mathrm{SD}, \mathrm{mL} / 100 \mathrm{~mL} / \mathrm{min}$ & $36.9 \pm 24.2$ & $41.2 \pm 30.0$ & 0.014 \\
\hline $\mathrm{BF}_{\text {max }}$, mean $\pm \mathrm{SD}, \mathrm{mL} / 100 \mathrm{~mL} / \mathrm{min}$ & $56.4 \pm 31.7$ & $64.6 \pm 8.7$ & 0.071 \\
\hline $\mathrm{BV}$, mean $\pm \mathrm{SD}, \mathrm{mL} / 100 \mathrm{~mL} / \mathrm{min}$ & $10.4 \pm 9.4$ & $11.4 \pm 9.7$ & 0.437 \\
\hline $\mathrm{MTT}$, mean $\pm \mathrm{SD}, \mathrm{s}$ & $14.9 \pm 4.4$ & $11.4 \pm 4.3$ & $<0.001$ \\
\hline \multicolumn{4}{|l|}{ Adenocarcinoma $(\mathrm{n}=22)$} \\
\hline $\mathrm{BF}$, mean $\pm \mathrm{SD}, \mathrm{mL} / 100 \mathrm{~mL} / \mathrm{min}$ & $31.3 \pm 14.5$ & $35.3 \pm 17.7$ & 0.050 \\
\hline $\mathrm{BF}_{\max }$, mean $\pm \mathrm{SD}, \mathrm{mL} / 100 \mathrm{~mL} / \mathrm{min}$ & $50.5 \pm 20.3$ & $83.5 \pm 19.3$ & 0.049 \\
\hline $\mathrm{BV}$, mean $\pm \mathrm{SD}, \mathrm{mL} / 100 \mathrm{~mL} / \mathrm{min}$ & $10.8 \pm 10.3$ & $12.0 \pm 10.2$ & 0.291 \\
\hline $\mathrm{MTT}$, mean $\pm \mathrm{SD}, \mathrm{s}$ & $15.2 \pm 4.7$ & $12.3 \pm 4.4$ & 0.002 \\
\hline \multicolumn{4}{|l|}{ Squamous cell carcinoma $(\mathrm{n}=8)$} \\
\hline $\mathrm{BF}$, mean $\pm \mathrm{SD}, \mathrm{mL} / 100 \mathrm{~mL} / \mathrm{min}$ & $33.1 \pm 16.7$ & $36.4 \pm 16.8$ & 0.401 \\
\hline $\mathrm{BF}_{\max }$, mean $\pm \mathrm{SD}, \mathrm{mL} / 100 \mathrm{~mL} / \mathrm{min}$ & $46.2 \pm 18.6$ & $50.6 \pm 19.4$ & 0.263 \\
\hline $\mathrm{BV}$, mean $\pm \mathrm{SD}, \mathrm{mL} / 100 \mathrm{~mL} / \mathrm{min}$ & $6.4 \pm 3.4$ & $6.8 \pm 3.2$ & 0.889 \\
\hline $\mathrm{MTT}$, mean $\pm \mathrm{SD}, \mathrm{s}$ & $16.1 \pm 3.2$ & $11.5 \pm 2.9$ & 0.017 \\
\hline \multicolumn{4}{|l|}{ Large-cell carcinoma $(\mathrm{n}=7)$} \\
\hline $\mathrm{BF}$, mean $\pm \mathrm{SD}, \mathrm{mL} / 100 \mathrm{~mL} / \mathrm{min}$ & $58.8 \pm 15.8$ & $66.5 \pm 20.9$ & 0.237 \\
\hline $\mathrm{BF}_{\max }$, mean $\pm \mathrm{SD}, \mathrm{mL} / 100 \mathrm{~mL} / \mathrm{min}$ & $78.8 \pm 53.5$ & $82.3 \pm 105.6$ & 0.128 \\
\hline $\mathrm{BV}$, mean $\pm \mathrm{SD}, \mathrm{mL} / 100 \mathrm{~mL} / \mathrm{min}$ & $13.4 \pm 10.4$ & $14.4 \pm 12.2$ & 0.866 \\
\hline $\mathrm{MTT}$, mean $\pm \mathrm{SD}, \mathrm{s}$ & $35.5 \pm 21.4$ & $8.6 \pm 4.7$ & 0.018 \\
\hline
\end{tabular}

BF indicates blood flow; CT, computed tomography; BV, blood volume; MTT, mean transit time.

coworkers ${ }^{36}$ recently showed that the comparably high BF of LCC shows the best response of all NSCLC subtypes to antiangiogenic treatment. We found significant differences in pulmonary $\mathrm{PI}_{\max }$ using a tumor volume cutoff of $3.5 \mathrm{~cm}^{3}$, with higher pulmonary $\mathrm{PI}_{\max }$ in larger tumors. This implies an increase in pulmonary blood supply with growing tumor size. Our findings correspond reasonably to the results of the study by Kiessling et $\mathrm{al}^{37}{ }^{37}$ who found lower perfusion in tumors larger than $50 \mathrm{~mm}^{3}$, using a bronchial arterial system analysis. Tumor volume certainly reflects the actual tumor size more exactly than does the maximum diameter alone. Given an ellipsoid-shaped tumor, it is obvious that the maximum diameter might not correctly reflect the actual tumor volume. ${ }^{38}$ This may be one likely reason we could not find a discriminative cutoff for the maximum tumor diameter.

In 1 of the first studies on this topic, Milne ${ }^{16}$ demonstrated that the pulmonary circulation supplies the surface of the tumor, whereas the central portion is supplied by the bronchial artery system. With increasing tumor volume, hypoxia may occur in the central parts of the tumor as a result of insufficient blood supply due to an increasing diffusion distance between blood vessels and tumor cells, ${ }^{26,39}$ explaining the need to recruit additional blood vessels from the pulmonary system,

TABLE 3. CT Perfusion Parameters Stratified by NSCLC Subtypes

\begin{tabular}{|c|c|c|c|c|c|c|c|}
\hline Parameter & $\mathrm{AC}(\mathrm{n}=22)$ & $\operatorname{SCC}(n=8)$ & $\operatorname{LCC}(n=7)$ & $P$ & AC vs SCC & AC vs LCC & SCC vs LCC \\
\hline $\begin{array}{l}\text { BF bronchial, mean } \pm \mathrm{SD} \text {, } \\
\mathrm{mL} / 100 \mathrm{~mL} / \mathrm{min}\end{array}$ & $35.3 \pm 17.7$ & $36.4 \pm 16.8$ & $66.5 \pm 20.9$ & 0.002 & 0.479 & 0.024 & 0.237 \\
\hline $\begin{array}{l}\text { BF pulmonary, mean } \pm \mathrm{SD} \text {, } \\
\mathrm{mL} / 100 \mathrm{~mL} / \mathrm{min}\end{array}$ & $31.3 \pm 14.5$ & $33.1 \pm 16.7$ & $58.8 \pm 15.8$ & 0.033 & 0.044 & 0.018 & 0.176 \\
\hline $\begin{array}{l}\mathrm{BV} \text { bronchial, mean } \pm \mathrm{SD} \\
\mathrm{mL} / 100 \mathrm{~mL} / \mathrm{min}\end{array}$ & $12.0 \pm 10.2$ & $6.8 \pm 3.2$ & $14.4 \pm 12.2$ & 0.001 & 0.051 & 0.499 & 0.128 \\
\hline $\begin{array}{l}\mathrm{BV} \text { pulmonary, mean } \pm \mathrm{SD} \text {, } \\
\mathrm{mL} / 100 \mathrm{~mL} / \mathrm{min}\end{array}$ & $10.8 \pm 10.3$ & $6.4 \pm 3.4$ & $13.4 \pm 10.4$ & 0.007 & 0.208 & 0.735 & 0.091 \\
\hline MTT pulmonary, mean $\pm \mathrm{SD}, \mathrm{s}$ & $15.2 \pm 4.7$ & $16.3 \pm 3.1$ & $12.9 \pm 4.8$ & 0.007 & 0.717 & 0.326 & 0.168 \\
\hline MTT bronchial, mean $\pm \mathrm{SD}, \mathrm{s}$ & $12.3 \pm 4.4$ & $11.5 \pm 2.9$ & $8.6 \pm 4.7$ & 0.288 & 0.985 & 0.236 & 0.267 \\
\hline Pulmonary $\mathrm{PI}_{\text {mean }}$, mean $\pm \mathrm{SD}$ & $0.4 \pm 0.1$ & $0.5 \pm 0.1$ & $0.5 \pm 0.1$ & 0.150 & 0.475 & 0.750 & 0.824 \\
\hline Pulmonary $\mathrm{PI}_{\max }$ mean $\pm \mathrm{SD}$ & $0.4 \pm 0.1$ & $0.5 \pm 0.04$ & $0.5 \pm 0.08$ & $<0.001$ & 0.001 & 0.001 & 0.660 \\
\hline Volume, median (range), $\mathrm{cm}^{3}$ & $8.9(0.8-156)$ & $16.3(2.62-153.0)$ & $13.2(3.3-32.2)$ & 0.622 & 0.168 & 0.328 & 0.269 \\
\hline Diameter, median (range), mm & $4.2(1.0-5.1)$ & $3.4(2.0-8.4)$ & $4.6(2.8-4.9)$ & 0.565 & 0.103 & 0.942 & 0.195 \\
\hline
\end{tabular}

As bronchial PI being defined as 1-pulmonary PI, bronchial PI is not shown in this table.

AC indicates adenocarcinoma; BF, blood flow; BV, blood volume; CT, computed tomography; LCC, large-cell carcinoma; MTT, mean transit time; NSCLC, non--small cell lung cancer; PI, perfusion index; SCC, squamous cell carcinoma. 
which carries deoxygenated blood but has a much larger cross-sectional area than the bronchial system does. Hence, our results provide further evidence for the theory that NSCLCs have a tendency to recruit more new blood vessels from the pulmonary system than from the bronchial system to grow. It however remains unclear if growing tumors might not just surround pulmonary vessels recruiting them, rather than constituting real angiogenesis originating from pulmonary vessels.

If interventional endovascular therapy is contemplated in an NSCLC patient, knowledge about the $\mathrm{PI}_{\max }$ may provide guidance for choosing a transbronchial or a transpulmonary approach. Transarterial chemoembolization has been used successfully for years for the treatment of hepatic malignancies ${ }^{40}$ and has recently gained attention as a minimally invasive approach for treating NSCLC. ${ }^{41}$ Recent studies demonstrated that the transpulmonary chemoembolization of different malignant lung lesions yielded a favorable tumor response without major complications. ${ }^{41-43}$ It is now considered a well-tolerated palliative treatment option in patients with lung metastases. ${ }^{44}$ However, today, no clear recommendation exists in which case a transpulmonary or transbronchial approach should be attempted. In this context, our results would suggest a preferred transpulmonary approach in tumors larger than $3.5 \mathrm{~cm}^{3}$, especially in LCC and SCC, and a transbronchial approach in tumors smaller than $3.5 \mathrm{~cm}^{3}$, especially in AC.

Although the response evaluation criteria in solid tumors were developed to assess tumoral response to cytotoxic agents, these may not properly reflect tumoral changes because of new antiangiogenic drugs. ${ }^{7,45}$ Computed tomography perfusion might represent a clinical tool to assess the "behavior" of tumoral angiogenesis under targeted antiangiogenic therapy. This concept has been addressed by several previous studies. ${ }^{9,15,46-48}$ Tacelli et al ${ }^{19,36}$ showed a significant reduction in total tumor vascular volume during treatment with chemotherapy using anti-vascular endothelial growth factor drugs. Fraioli et a $1^{19,36}$ demonstrated a significant difference in $\mathrm{BF}$ and $\mathrm{BV}$ in $\mathrm{LCC}$ patients for partial response and progressive disease. Although we did not address therapeutic effects, the results of our study indicate that both histological subtype and tumor volume likely should be taken into account when assessing tumoral treatment response using CTP. Further studies are needed to address treatment-induced effects on either blood supply, to define a potential role of pulmonary and/or bronchial CTP assessment for therapy monitoring.

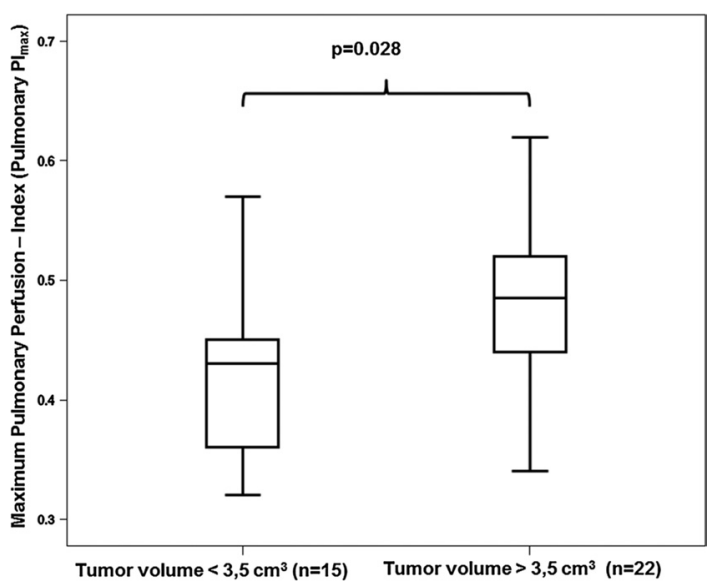

FIGURE 5. Pulmonary $\mathrm{PI}_{\max }$ in all tumors, using a volume cutoff of $3.5 \mathrm{~cm}^{3}$. $\mathrm{PI}_{\max }$ indicates maximum perfusion index.

Because we applied a tube voltage of $100 \mathrm{kV}$ and found no bothersome beam-hardening, we believe that the voltage might even be reduced further for CTP studies, especially concerning the lung, to increase the sensitivity to the contrast agent and to reduce both dose exposure and contrast agent administered to the patient.

Our study has several limitations. Our approach was not able to directly and independently measure the contribution of the pulmonary and bronchial vasculature to the overall tumor perfusion. This was, however, not our goal because we aimed at identifying differences in CTP parameters depending on the type of the arterial input ROI placement. Most of our subjects had AC, which means that the overall results for NSCLC were dependent largely upon this histological subtype. This, however, does not affect the subanalyses carried out for all histological subtypes, although LCC and SCC numbers were comparably small. Tumor stage was somewhat skewed according to NSCLC subtypes, with LCC patients having by trend more advanced stages. This may also have influenced our results. As well, most of the reported tumor stages were clinical and not pathological.

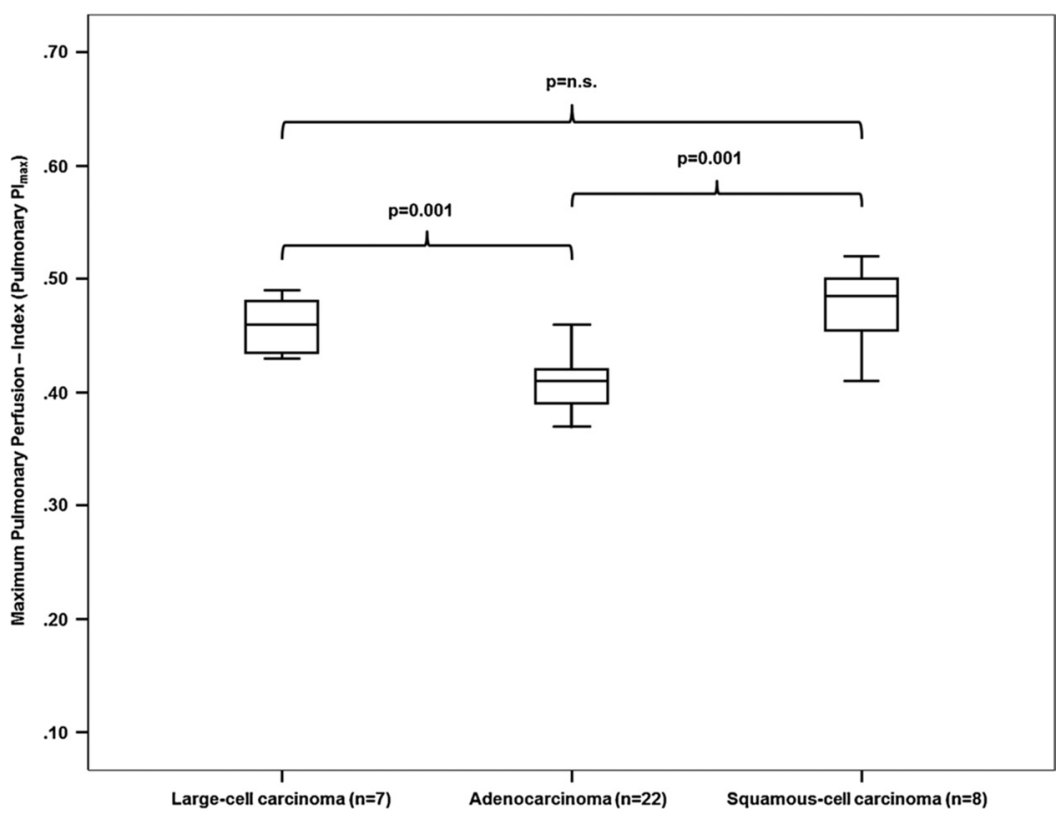

FIGURE 4. Pulmonary $\mathrm{PI}_{\max }$ of non-small cell lung cancer subtypes. $\mathrm{PI}_{\max }$ indicates maximum perfusion index. 


\section{CONCLUSIONS}

Our study is the first to address differences in the assessment of NSCLC and its subtypes by CTP, using both a pulmonary and a bronchial vasculature approach. The known dual blood supply of NSCLC is reflected by differences in CTP parameters and depends both on tumor size and histological subtype. A generally higher bronchialderived than pulmonary-derived $\mathrm{BF}$ was found in $\mathrm{AC}$, as well as both higher pulmonary-derived and bronchial-derived BF in LCC compared with other subtypes. Larger tumors have a higher pulmonary than bronchial contribution to their blood supply compared with smaller tumors. Therefore, a CTP analysis of lung cancer, either in a primary setup or for the assessment of therapy response, should consider that parameters cannot simply be translated between NSCLC subtypes as well as between pulmonary and bronchial approaches. Furthermore, with growing or regressing tumors, the ratio of pulmonary and bronchial blood supply as expressed by CTP parameters might vary. This has to be accounted for when analyzing NSCLC with either a pulmonary or a bronchial vasculature approach.

\section{REFERENCES}

1. American Cancer Society. Cancer Facts \& Figures 2014. Atlanta, GA: American Cancer Society; 2014

2. Kaira K, Tomizawa Y, Yoshino R, et al. Phase II study of oral S-1 plus cisplatin with bevacizumab for advanced non-squamous non-small cell lung cancer. Lung Cancer. 2013;82:103-108.

3. Pfister DG, Johnson DH, Azzoli CG, et al. American Society of Clinical Oncology treatment of unresectable non-small-cell lung cancer guideline: update 2003. J Clin Oncol. 2004;22:330-353.

4. Di Costanzo F, Mazzoni F, Micol Mela M, et al. Bevacizumab in non-small cell lung cancer. Drugs. 2008;68:737-746.

5. Drevs J, Schneider V. The use of vascular biomarkers and imaging studies in the early clinical development of anti-tumour agents targeting angiogenesis. J Intern Med. 2006;260:517-529.

6. Ng QS, Goh V. Angiogenesis in non-small cell lung cancer: imaging with perfusion computed tomography. J Thorac Imaging. 2010;25:142-150.

7. Ratain MJ, Eckhardt SG. Phase II studies of modern drugs directed against new targets: if you are fazed, too, then resist RECIST. J Clin Oncol. 2004;22: 4442-4445.

8. Goh V, Ng QS, Miles K. Computed tomography perfusion imaging for therapeutic assessment: has it come of age as a biomarker in oncology? Invest Radiol. 2012; 47:2-4.

9. Fraioli F, Anzidei M, Zaccagna F, et al. Whole-tumor perfusion CT in patients with advanced lung adenocarcinoma treated with conventional and antiangiogenetic chemotherapy: initial experience. Radiology. 2011;259:574-582.

10. Jiang T, Kambadakone A, Kulkarni NM, et al. Monitoring response to antiangiogenic treatment and predicting outcomes in advanced hepatocellular carcinoma using image biomarkers, CT perfusion, tumor density, and tumor size (RECIST). Invest Radiol. 2012;47:11-17.

11. Ng QS, Goh V, Klotz E, et al. Quantitative assessment of lung cancer perfusion using MDCT: does measurement reproducibility improve with greater tumor volume coverage? AJR Am J Roentgenol. 2006;187:1079-1084.

12. Reiner CS, Goetti R, Eberli D, et al. CT perfusion of renal cell carcinoma: impact of volume coverage on quantitative analysis. Invest Radiol. 2012;47:33-40.

13. Morsbach F, Pfammatter T, Reiner CS, et al. Computed tomographic perfusion imaging for the prediction of response and survival to transarterial radioembolization of liver metastases. Invest Radiol. 2013;48:787-794.

14. Choi SH, Chung JW, Kim HC, et al. The role of perfusion CT as a follow-up modality after transcatheter arterial chemoembolization: an experimental study in a rabbit model. Invest Radiol. 2010;45:427-436.

15. Cyran CC, von Einem JC, Paprottka PM, et al. Dynamic contrast-enhanced computed tomography imaging biomarkers correlated with immunohistochemistry for monitoring the effects of sorafenib on experimental prostate carcinomas. Invest Radiol. 2012;47:49-57.

16. Milne EN. Circulation of primary and metastatic pulmonary neoplasms: a postmortem microarteriographic study. Am J Roentgenol Radium Ther Nucl Med. 1967;100:603-619.

17. Goetti R, Leschka S, Desbiolles L, et al. Quantitative computed tomography liver perfusion imaging using dynamic spiral scanning with variable pitch: feasibility and initial results in patients with cancer metastases. Invest Radiol. 2010;45: 419-426.
18. Bisdas S, Baghi M, Smolarz A, et al. Quantitative measurements of perfusion and permeability of oropharyngeal and oral cavity cancer, recurrent disease, and associated lymph nodes using first-pass contrast-enhanced computed tomography studies. Invest Radiol. 2007;42:172-179.

19. Tacelli N, Santangelo T, Scherpereel A, et al. Perfusion CT allows prediction of therapy response in non-small cell lung cancer treated with conventional and anti-angiogenic chemotherapy. Eur Radiol. 2013;23:2127-2136.

20. Miles KA, Griffiths MR, Fuentes MA. Standardized perfusion value: universal $\mathrm{CT}$ contrast enhancement scale that correlates with FDG PET in lung nodules. $R a$ diology. 2001;220:548-553.

21. Li Y, Yang ZG, Chen TW, et al. First-pass perfusion imaging of solitary pulmonary nodules with 64-detector row CT: comparison of perfusion parameters of malignant and benign lesions. Br J Radiol. 2010;83:785-790.

22. Yuan X, Zhang J, Quan C, et al. Differentiation of malignant and benign pulmonary nodules with first-pass dual-input perfusion CT. Eur Radiol. 2013;23: 2469-2474.

23. Cronin P, Dwamena BA, Kelly AM, et al. Solitary pulmonary nodules: metaanalytic comparison of cross-sectional imaging modalities for diagnosis of malignancy. Radiology. 2008;246:772-782.

24. Shi J, Schmid-Bindert G, Fink C, et al. Dynamic volume perfusion CT in patients with lung cancer: baseline perfusion characteristics of different histological subtypes. Eur J Radiol. 2013;82:e894-e900.

25. Tacelli N, Remy-Jardin M, Copin MC, et al. Assessment of non-small cell lung cancer perfusion: pathologic-CT correlation in 15 patients. Radiology. 2010; 257:863-871.

26. Spira D, Neumeister H, Spira SM, et al. Assessment of tumor vascularity in lung cancer using volume perfusion CT (VPCT) with histopathologic comparison: a further step toward an individualized tumor characterization. J Comput Assist Tomogr. 2013;37:15-21.

27. Huellner MW, Collen TD, Gut P, et al. Multiparametric PET/CT-perfusion does not add significant additional information for initial staging in lung cancer compared with standard PET/CT. EJNMMI Res. 2014;4:6.

28. Lee SM, Lee HJ, Kim JI, et al. Adaptive 4D volume perfusion CT of lung cancer: effects of computerized motion correction and the range of volume coverage on measurement reproducibility. AJR Am J Roentgenol. 2013;200:W603-W609.

29. Ippolito D, Capraro C, Casiraghi A, et al. Quantitative assessment of tumour associated neovascularisation in patients with liver cirrhosis and hepatocellular carcinoma: role of dynamic-CT perfusion imaging. Eur Radiol. 2012;22:803-811.

30. Sauter AW, Winterstein S, Spira D, et al. Multifunctional profiling of non-small cell lung cancer using 18F-FDG PET/CT and volume perfusion CT. $\mathrm{J} \mathrm{Nucl}$ Med. 2012;53:521-529.

31. Ng CS, Chandler AG, Wei W, et al. Reproducibility of perfusion parameters obtained from perfusion CT in lung tumors. AJR Am J Roentgenol. 2011;197: $113-121$.

32. Travis WD. Lung tumours with neuroendocrine differentiation. Eur $J$ Cancer. 2009;45(suppl 1):251-266.

33. Grand B, Cazes A, Mordant P, et al. High grade neuroendocrine lung tumors: pathological characteristics, surgical management and prognostic implications. Lung Cancer. 2013;81:404-409.

34. Travis W, Brambilla E, Muller-Hermelink H, et al. Tumours of the lung, pleura thymus and heart. Lyon, France: International Agency for Research on Cancer (IARC) Press; 2004:9-124.

35. Rossi G, Mengoli MC, Cavazza A, et al. Large cell carcinoma of the lung: clinically oriented classification integrating immunohistochemistry and molecular biology. Virchows Arch. 2014;464:61-68.

36. Fraioli F, Anzidei M, Serra G, et al. Whole-tumour CT-perfusion of unresectable lung cancer for the monitoring of anti-angiogenetic chemotherapy effects. $\mathrm{Br} J$ Radiol. 2013;86:20120174.

37. Kiessling F, Boese J, Corvinus C, et al. Perfusion CT in patients with advanced bronchial carcinomas: a novel chance for characterization and treatment monitoring? Eur Radiol. 2004;14:1226-1233.

38. Tsai CH, Lin CM, Hsieh CC, et al. Tumor volume is a better prognostic factor than greatest tumor diameter in stage Ia non-small cell lung cancer. Thorac Cardiovasc Surg. 2006;54:537-543.

39. Nelson AR, Fingleton B, Rothenberg ML, et al. Matrix metalloproteinases: biologic activity and clinical implications. J Clin Oncol. 2000;18:1135-1149.

40. Gonsalves CF, Brown DB. Chemoembolization of hepatic malignancy. Abdom Imaging. 2009;34:557-565.

41. Vogl TJ, Shafinaderi M, Zangos S, et al. Regional chemotherapy of the lung: transpulmonary chemoembolization in malignant lung tumors. Semin Intervent Radiol. 2013;30:176-184.

42. Vogl TJ, Wetter A, Lindemayr S, et al. Treatment of unresectable lung metastases with transpulmonary chemoembolization: preliminary experience. Radiology. 2005;234:917-922. 
43. Lindemayr S, Lehnert T, Korkusuz H, et al. Transpulmonary chemoembolization: a novel approach for the treatment of unresectable lung tumors. Tech Vasc Interv Radiol. 2007;10:114-119.

44. Vogl TJ, Herzog C, Zangos S, et al. Palliative treatment of primary lung tumors with transpulmonary chemoembolization (TPCE). Rofo. 2007;179: 300-307.

45. Eisenhauer EA, Therasse P, Bogaerts J, et al. New response evaluation criteria in solid tumours: revised RECIST guideline (version 1.1). Eur J Cancer. 2009;45: $228-247$.
46. Wang J, Wu N, Cham MD, et al. Tumor response in patients with advanced nonsmall cell lung cancer: perfusion CT evaluation of chemotherapy and radiation therapy. AJR Am J Roentgenol. 2009;193:1090-1096.

47. Lind JS, Meijerink MR, Dingemans AM, et al. Dynamic contrast-enhanced CT in patients treated with sorafenib and erlotinib for non-small cell lung cancer: a new method of monitoring treatment? Eur Radiol. 2010;20:2890-2898.

48. Ren Y, Fleischmann D, Foygel K, et al. Antiangiogenic and radiation therapy: early effects on in vivo computed tomography perfusion parameters in human colon cancer xenografts in mice. Invest Radiol. 2012;47:25-32. 